\title{
Automatic Modulation Recognition of both Digital and Analog Communication Signals
}

\author{
Tiger Sun, Jiakuan Jia and Guojun $\mathrm{Yu}^{*}$ \\ Faculty of Civil Engineering and Mechanics, Jiangsu University, Zhenjiang, Jiangsu, 212013 China \\ ${ }^{*}$ Corresponding author
}

\begin{abstract}
Six feature parameters P1-P6 based on the first statistical moments of the received signals were proposed for automatically classify the modulation of digital and analog communication signal, which were calculated by signal processing methods. The calculation process is simpler than witch traditional algorithm, but also this process is suitable for real time online. The automatic modulation recognition algorithm was also given based on the decision-theoretic approach, which was presented in this work. With 6 Thresholds T1-T6, the 7 kinds of digital and analog communication signal were recognized with an average success rate $\geq 98 \%$, including $2 \mathrm{ASK}$, 4ASK, 2FSK, 4FSK, AM, DSO and 16QAM, by the MATLAB simulation.
\end{abstract}

Keywords-automatic modulation recognition; digital and analog signals; the first statistical moment; feature parameters

\section{INTRODUCTION}

The automatic detection and recognition of digital and analog communication signals modulation method is a relatively new research direction in the field of signal analysis. Modulation method is an important parameter of signal feature. With the rising of electronic countermeasure technology, the urgent need for the automatic identification technology research of digital communication signals modulation way, it is widely used in: signal confirmation, interference identification, radio detection, electronic countermeasures, signal monitoring and threat analysis, etc [1-5].

Through the method of judgment, this paper puts forward an algorithm whose way of modulation can be recognized automatically 2ASK, 4ASK, 2FSK、4FSK, 16QAM, AM, DSB and so on [6-12]. The process of implementing recognition algorithm is given to distinguish the commonly used digital modulation style. In addition, a relatively few parameters are selected, it's easy to extract with small amount of calculation and strong robustness.

\section{THE CHARACTERISTIC PARAMETERS OF THE MODULATION SIGNALS}

We assume that does not require the synchronous cold-elements in the signal receiver. When receiving the 6 kinds of digital and analog communication signals in the introduction, extracting the feature parameters are shown in TABLE I.

The first characteristic parameter $\mathrm{P} 1$ is defined as the unimodal number of the received signal spectrum. When we receive the signal, carries on the Fourier transform. Within a narrow spectrum, if the difference value between a component and other components is greater than a certain threshold, argues that appear a single-peak; if there are difference values between two components and other components greater than the threshold, and there is a certain distance between the two components, argues that there are two peaks within the spectrum, and so on. Characteristic parameters distinguish between the 2ASK, 4ASK and other signals, because 2ASK, 4ASK signals are single-peak.

TABLE I. THE FEATURE PARAMETERS OF DIGITAL AND ANALOG COMMUNICATION SIGNALS

\begin{tabular}{|c|c|}
\hline Signals & Feature Parameters \\
\hline $\mathrm{P} 1$ & $\mathrm{P}_{1}=n$ \\
\hline $\mathrm{P} 2$ & $\mathrm{P}_{2}=\sqrt{\frac{1}{N}\left[\sum_{i=1}^{N} A_{\mathrm{cn}}^{2}(i)\right]-\left[\frac{1}{N} \sum_{i=1}^{N} \mid A_{c n}(i)\right]^{2}}$ \\
\hline $\mathrm{P} 3$ & $\mathrm{P}_{3}=\frac{1}{N} \max \left|\mathrm{FFT}\left[A_{c n}(i)\right]^{2}\right|$ \\
\hline $\mathrm{P} 4$ & $\mathrm{P}_{4}=\frac{1}{M} \sum_{i=1}^{M}|| \mathrm{F}(i)\left|-\frac{1}{M} \sum_{k=1}^{M}\right| \mathrm{F}(k) \mid$ \\
\hline $\mathrm{P} 5$ & $\mathrm{P}_{5}=\frac{\sigma^{2}}{\mu^{2}}$ \\
\hline $\mathrm{P} 6$ & $\mathrm{P}_{6}=\frac{1}{M} \sum_{i=1}^{M}\left|\phi_{c}(i)\right|-\frac{1}{M} \sum_{k=1}^{M}\left|\phi_{c}(k)\right|$ \\
\hline
\end{tabular}

Among them: $\mathrm{N}$ is the total number of sampling, Acn(i) normalized instantaneous amplitude zero center, there is

$$
\left\{\begin{array}{l}
A_{c n}(i)=A_{n}(i)-1 \\
A_{n}(i)=\frac{A(i)}{\mathrm{m}_{a}}
\end{array}\right.
$$

where $A(i)$ is the receiving signal amplitude of the moment $i$, when $\mathrm{N}$ is the total number of sampling, may be achieved through the general signal processing technology, $m_{a}$ is the average of $A(i)$, and defined as:

$$
\mathrm{m}_{a}=\frac{1}{N} \sum_{i=1}^{N} A(i)
$$

Characteristic parameters $\mathrm{P} 3$ is mainly used to distinguish between A 2FSK, 4FSK and other signals, because the envelope of $2 \mathrm{FSK}$ and $4 \mathrm{FSK}$ signal are 
constant, there is $\mathrm{P}_{3}=0$, and the rest class of signal are not zero.

$$
\mathrm{F}(i)=\frac{1}{\mathrm{~F}_{s}}\left[f(i)-\frac{1}{M} \sum_{k=1}^{M} f(k)\right]
$$

where $f(i)$ is the received signal frequency at the moment $i$, when sampling a total of $\mathrm{N}$.

For 2ASK signal, the absolute value of its extent is constant, so the characteristic parameters of $P_{2}=0$. But it's not a constant for 4ASK signal, there is $\mathrm{P}_{2} \neq 0$, so the feature parameters are used to distinguish the signal $2 \mathrm{ASK}$ and 4ASK can obtain an ideal result.

Characteristic parameters $P_{3}$ is mainly used to distinguish between A 2FSK, 4FSK and other signals, because the envelope of 2FSK and 4FSK signal are constant, there is $\mathrm{P} 3=0$, and the rest class of signal are not zero. The characteristic parameter $P_{4}$ is mainly used to distinguish between the 2FSK and 4FSK signal. The 2FSK signal $f(i)$ contains two discrete values, so $P_{4}=0$. The 4FSK signal $f(i)$ contains four discrete values, so $\mathrm{P}_{4} \neq 0$.

The fifth characteristic parameter P5 mainly reflects the changeable extent of signal envelope.

No noise in the ideal, the 2PSK signal due to its instantaneous phase $\Phi(i)$ with two discrete values $0 、 \pi$. Therefore, after taking the absolute value in the center of zero, the two discrete values become $\pi / 2$, are constant, and $\mathrm{P}_{6}=0$; But the instantaneous phase of 4PSK signal contains four discrete values. Therefore, after taking the absolute value in the center of zero, the $\Phi(i)$ will have two discrete values, namely, $\mathrm{P}_{6}=0$. Therefore, we can distinguish between 2PSK and 4PSK signals through the characteristic parameters $P_{6}$.

\section{RECOGNITION ALGORITHM}

The method of modulation in time domain and frequency domain have different characteristics: in the time domain has permanently envelope signals and constant envelope; look on the spectrum, there are residual carrier signal and no residual carrier signal. Based on decision theory, the modulation signal in section 2 of the characteristics of all kinds of characteristic parameters are shown in the modulation mode automatic identification algorithm flow chart including threshold T1-T6 as shown in Table II.

TABLE II. THRESHOLD VALUE AND SIGNAL TO NOISE RATIO OF CHARACTERISTIC PARAMETERS

\begin{tabular}{|c|c|c|c|}
\hline Signals & Threshold & Threshold value & $\begin{array}{c}\text { Noise } \\
\text { ratio }\end{array}$ \\
\hline P1 & T1 & 1 & $\geq-1 \mathrm{~dB}$ \\
\hline P2 & T2 & 0.25 & $\geq-1 \mathrm{~dB}$ \\
\hline P3 & T3 & 0.87 & $\geq 3 \mathrm{~dB}$ \\
\hline P4 & T4 & 0.82 & $\geq-5 \mathrm{~dB}$ \\
\hline P5 & T5 & 0.88 & $\geq 5 \mathrm{~dB}$ \\
\hline P6 & T6 & 0.82 & $\geq-15 \mathrm{~dB}$ \\
\hline
\end{tabular}

\section{IDENTIFY VALIDATION}

To evaluate the effectiveness of automatic identification and test method of digital and analog signals mentioned in this article, the threshold value of each characteristic parameters can be determined by MATLAB simulation. In this method of modulation, the 0,1 randomly generated sequence use the zero-mean Gaussian white noise. Digital baseband signal waveform use rectangular pulse, the number of samples taken 4000 , the carrier frequency taken $100 \mathrm{KHz}, \mathrm{SNR}$ taken from $-5 \sim 20 \mathrm{~dB}$. As shown in Figure I, there are fifth characteristic parameter $P_{5}$ and the simulation graphics of SNR. From the above conclusions: no matter in the type, quantity, minimum signal-to-noise ratio, and the average recognition rate of the pending identification signal, the performance of the proposed recognition algorithm is superior to the general algorithm.

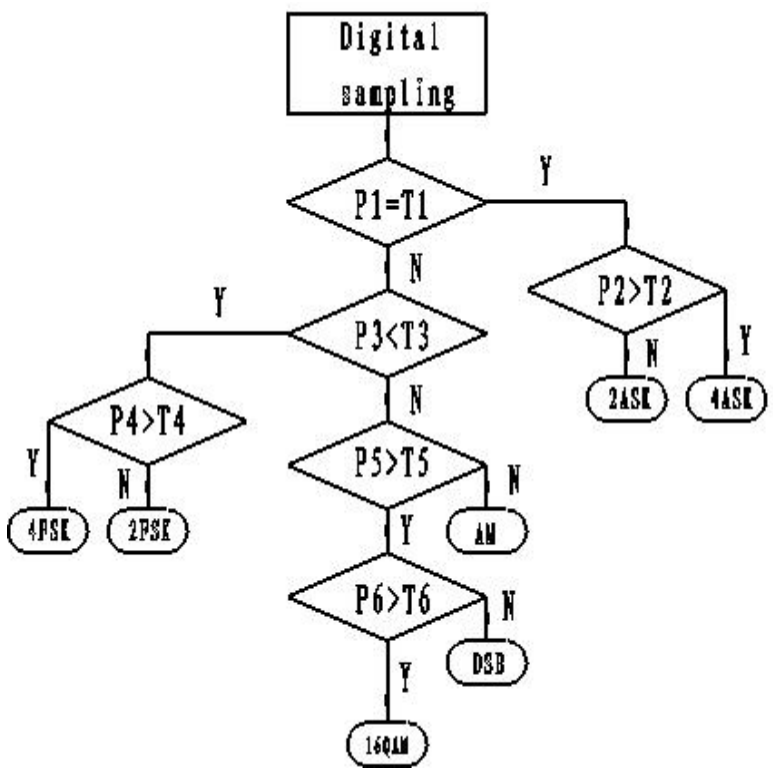

FIGURE I. AUTOMATIC MODULATION RECOGNITION ALGORITHM FLOW CHART

\section{CONCLUSIONS}

Based on the Decision Laws, the signal instantaneous amplitude, instantaneous phase and instantaneous rate on the basis of six characteristic parameters P1-6. The simulation results show that when the $\mathrm{SNR} \geq 15 \mathrm{db}$, the average recognition rate of 7 kinds of signal can reach $99.25 \%$. When $S N R \geq 9 \mathrm{db}$, except $16 \mathrm{QAM}$ is $77.5 \%$, other signals' recognition rate remains $100 \%$; When $\mathrm{SNR} \geq 6 \mathrm{db}$, except 16QAM is $77.5 \%$, other signals' recognition rate remains more than $96 \%$. When $\mathrm{SNR} \geq 5 \mathrm{db}$, except $16 \mathrm{QAM}$, other signals' recognition rate keep more than $87 \%$. When $\mathrm{SNR} \geq 0 \mathrm{db}$, the recognition rate of $2 \mathrm{FSK}$ signal can reach $90 \%$.

\section{ACKNOWLEDGEMENTS}

This work was the supported by the Research Foundation for Advanced Talents of Jiangsu University (Grant No. 14JDG162 and 14JDG161), Natural Science Foundation of 
Jiangsu Province (Grant No.BK20140560) and the National Natural Science Foundation of China (Grant No.51508237).

\section{REFERENCES}

[1] K. Alexandr, F. Benoit, C. Stephane, etc. Identification of the manipulator stiffness model parameters in industrial environment. Mechanism and Machine Theory. 2015, Vol.90:P1-22.

[2] P. Vinay, G. Kamal. A hierarchical and adaptive mobile manipulator planner with base pose uncertainty. Autonomous Robots. 2015, Vol.39:P65-85.

[3] T. Sun, J. Wu, L. Chen. Investigation of New Properties of Magnetorheological Fluids with Carbon Nanotubes. Journal of Computational and Theoretical Nanoscience. 2015(11), Vol.12:P4143-4146.

[4] G. B. Bhusan, S. B. Kumar, S. Rana. Realtime performance analysis of different combinations of fuzzy-PID and bias controllers for a two degree of freedom electrohydraulic parallel manipulator. Robotics and Computer-Integrated Manufacturing. 2015, Vol.34:P62-69.

[5] G. Yu, C. Du, T. Sun*. Thermodynamic Behaviors of a kind of Self-Decoupling Magnetorheological Damper. Shock and Vibration. 2015, Article Number: 502747.

[6] T. Sun, X. Peng, S. Jiao, C. Xu, J. Li. Simulation of Particle Dynamics of Nano-Magnetorheological Materials in External Magnetic Fields. Journal of Computational and Theoretical Nanoscience. 2014(6), Vol.11:P1518-1523.

[7] B. Lee. Development of coaxial wire reduction gear for manipulator robots. Electronics Letters. 2015, Vol.51:P747-748.

[8] Q. Y. He, X. L. Gong, S. H. Xuan.Shear thickening of suspensions of porous silica nanoparticles. Journal of Materials Science. 2015(50): P 6041- 6049

[9] D. Z. Xu, L. Mi, Z. S. Wu. Energy damage detection strategy based on strain responses for long-span bridge structures. Journal of Bridge Engineering, ASCE, 2011(5), Vol.16:P644-652.

[10]J. Q. Yu, X. M. Dong, W. Wang. Prototype and test of a novel rotary magnetorheological damper based on helical flow. Smart Materials and Structures. 2016(2), Vol.25, Article ID: 025006.

[11] J. Zhang, C. Y. Wang, A. Sondipon. Reply to Comment on 'Molecular structure-dependent deformations in boron nitride nanostructures subject to an electrical field'. Journal of Physics D-Applied Physics. 2016(10), Vol.49:P44-65.

[12]D.H.Chen, K.Ushijima. Singular stresses due to adhesion defect on intersection line along which a semi-infinite thin plate is attached to an infinite thin plate by eigenfunction expansion method. Theoretical and Applied Fracture Mechanics. 2014, Vol.69: P 71-79. 